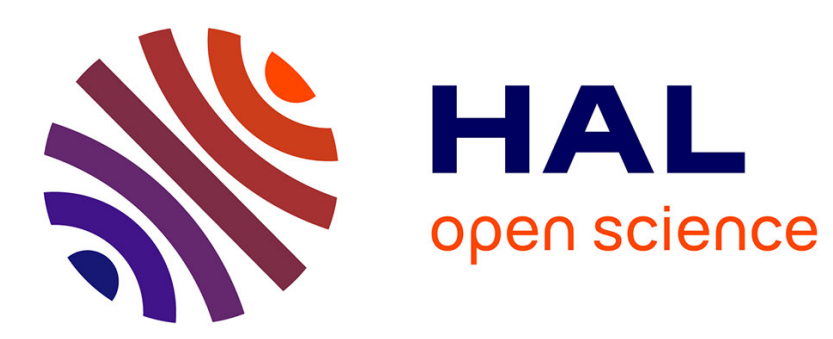

\title{
Etude en petites déformations de l'interaction entre une fondation superficielle et une conduite enterrée
}

\author{
Roger Frank, Olivier Thépot
}

\section{To cite this version:}

Roger Frank, Olivier Thépot. Etude en petites déformations de l'interaction entre une fondation superficielle et une conduite enterrée. Revue Européenne de Génie Civil, 2005, 9 (9-10), pp.1095-1109. 10.1080/17747120.2005.9692802 . hal-00557078

\section{HAL Id: hal-00557078 \\ https://hal.science/hal-00557078}

Submitted on 16 May 2018

HAL is a multi-disciplinary open access archive for the deposit and dissemination of scientific research documents, whether they are published or not. The documents may come from teaching and research institutions in France or abroad, or from public or private research centers.
L'archive ouverte pluridisciplinaire HAL, est destinée au dépôt et à la diffusion de documents scientifiques de niveau recherche, publiés ou non, émanant des établissements d'enseignement et de recherche français ou étrangers, des laboratoires publics ou privés. 


\title{
Etude en petites déformations de l'interaction entre une fondation superficielle et une conduite enterrée
}

\author{
Roger Frank* - Olivier Thépot**
}

* CERMES (ENPC-LCPC), 6-8 avenue Blaise Pascal, Cité Descartes

Champs-sur-Marne, F-77454 Marne-la-Vallée cedex

** EAUDEPARIS (Service MAC), 2 avenue de la Convention

F-94110 Arcueil

thepot@eaudeparis.fr

RÉSUMÉ. Cet article présente la modélisation par la méthode des éléments finis de l'interaction entre une fondation superficielle et une conduite enterrée. On compare une loi élastoplastique (critère de Mohr-Coulomb) à partie élastique linéaire avec une loi élastique non linéaire de type hyperbolique. On montre que la loi élastoplastique ne permet pas de représenter correctement à la fois le comportement de la fondation et celui de la conduite. En effet, le choix d'un module adapté à la prévision du tassement de la fondation entraîne une sous-estimation importante de la raideur du sol autour de la conduite et, par conséquent, une sur-estimation des contraintes dans la conduite. En revanche, la loi élastique non linéaire permet de représenter simultanément la raideur élevée du sol autour de la conduite et la diminution significative de raideur du sol directement sous la fondation. Le recours à l'élasticité non linéaire est donc intéressant dès lors qu'il est possible de déterminer la variation du module avec la déformation et si le chargement est monotone.

ABSTRACT. This paper presents the finite element modelling of the interaction between a shallow foundation and a buried pipe. An elastoplastic model with a linear elastic part and Mohr-Coulomb criterion is compared to a non-linear elastic hyperbolic model. It is shown that the elastoplastic model does not allow to represent properly the behaviour of both the foundation and the pipe. Indeed, the choice of a modulus adapted to the prediction of the settlement of the foundation leads to an important underestimation of the stiffness of the soil around the pipe and, consequently, to an overestimation of the stresses in the pipe. On the contrary, the non-elastic model is capable of representing both the high stiffness around the pipe and the significant decrease in stiffness directly below the foundation. The use of nonlinear elasticity is thus interesting, provided it is possible to determine the variation of the modulus with strain and if the loading in monotonous.

MOTS-CLÉS : interaction sol-structure, élasticité non linéaire, conduite enterrée.

KEYWORDS: soil-structure interaction, non linear elasticity, buried pipe. 


\section{Introduction}

La forte densité d'occupation du sous-sol urbain oblige à prendre en compte les interactions multiples entre ouvrages et, notamment, avec les réseaux existants. Les collecteurs d'assainissement, qui forment la trame la plus ancienne et encore la plus importante des réseaux enterrés, représentent un patrimoine économique considérable (plus de $30000 \mathrm{~km}$ d'ouvrages visitables en France) dont le coût d'entretien, de réhabilitation ou de renouvellement est en constante progression. Ces ouvrages, qui datent parfois du $\mathrm{XIX}^{\mathrm{e}}$ siècle, présentent une grande diversité de matériaux (maçonnerie le plus souvent) et de formes, et sont généralement faiblement enterrés. Situés dans des sous-sols urbains de plus en plus encombrés, les collecteurs sont soumis aux sollicitations agressives du trafic, qui a largement augmenté depuis le début du $\mathrm{XX}^{\mathrm{e}}$ siècle, et doivent subir les changements de leurs environnements proches : construction d'un parking ou élargissement d'une plateforme ferroviaire, par exemple. La faible résistance de la maçonnerie, notamment en traction, restreint les capacités d'adaptation de ces ouvrages dont la stabilité repose largement sur l'interaction avec le sol encaissant (Thépot, 2004).

Le projet de construction d'un ouvrage d'une certaine importance en milieu urbain est obligatoirement précédé d'une étude d'impact sur les ouvrages existants, qui peut déboucher sur des modifications notables du projet, éventuellement accompagnées de renforcement du ou des ouvrages concernés qui peut aller jusqu'au remplacement. Ces adaptations et les renforcements éventuels ont, bien entendu, un coût que les gestionnaires de réseaux n'hésitent pas à faire supporter au maître d'ouvrage du projet. Il n'est donc plus rare que des études «poussées », utilisant des méthodes de modélisation comme la technique des éléments finis, soient réalisées pour estimer l'impact d'un projet sur un grand collecteur. Ces études sont généralement demandées par les gestionnaires de réseaux, dont les niveaux d'exigences sont très élevés eu égard à la fonction "stratégique» de ces ouvrages, qui doivent fonctionner en permanence et rester étanches.

La méthode des éléments finis, de par sa capacité à prendre en compte les géométries les plus complexes et les différentes lois de comportement des matériaux et des interfaces, est un outil bien adapté à la modélisation des interactions multiples (Frank, 1999). Les modèles de comportement des massifs couramment utilisés dans les modèles d'éléments finis utilisent des lois élastoplastiques, dont la partie élastique est généralement linéarisée. Comme on s'intéresse au comportement en service des ouvrages, les zones plastiques sont d'étendues limitées et la plus grande partie du sol reste dans le domaine élastique. (Jardine et al., 1986) ont montré que, dans de nombreux cas, les déformations de la plus grande partie du sol restent inférieures à $0,1 \%$. Le choix du module élastique du sol a donc une grande importance pour la prévision des déplacements; cependant, la raideur des sols décroissant très rapidement avec la déformation, pratiquement d'un ordre de grandeur quand la déformation varie de $0,01 \%$ à $1 \%$, la détermination du module d'Young est délicate et le résultat de cette détermination est difficile à justifier. 
D'autre part, les modules obtenus avec les essais habituels (essai pressiométrique, œdométrique ou triaxial) correspondent à des déformations de quelques pour-cent, à l'exception notable des essais de propagation d'ondes. On ne peut donc pas les « introduire » directement dans un modèle aux éléments finis, mais on ne sait pas pour autant justifier théoriquement les corrections que l'on applique à ces modules. Le recours à une loi élastique non linéaire, éventuellement couplée avec un critère plastique, est une alternative intéressante qui améliore sensiblement la modélisation des sols dans le domaine des charges de service par rapport à une loi élastoplastique à élasticité linéaire (Burland, 1989). On présente, dans cette communication, une application d'un modèle élastique non linéaire à un problème d'interaction entre une fondation superficielle (semelle souple) et un collecteur enterré.

\section{Détermination du module du sol pour une loi élastoplastique avec partie élastique linéaire}

La figure 1 représente l'évolution du module sécant en fonction de la déformation de cisaillement que l'on pourrait obtenir avec un triaxial de précision (Homsi, 1986). L'allure de la courbe est celle d'un $\mathrm{S}$ inversé d'où l'appellation courbe en $\mathrm{S}$ que l'on donne couramment à la variation du module sécant avec la déformation. Le module maximum tangent à l'origine est égal à $200 \mathrm{MPa}$ et sa valeur est divisée par 12 (un ordre de grandeur) pour une déformation de $1 \%$.

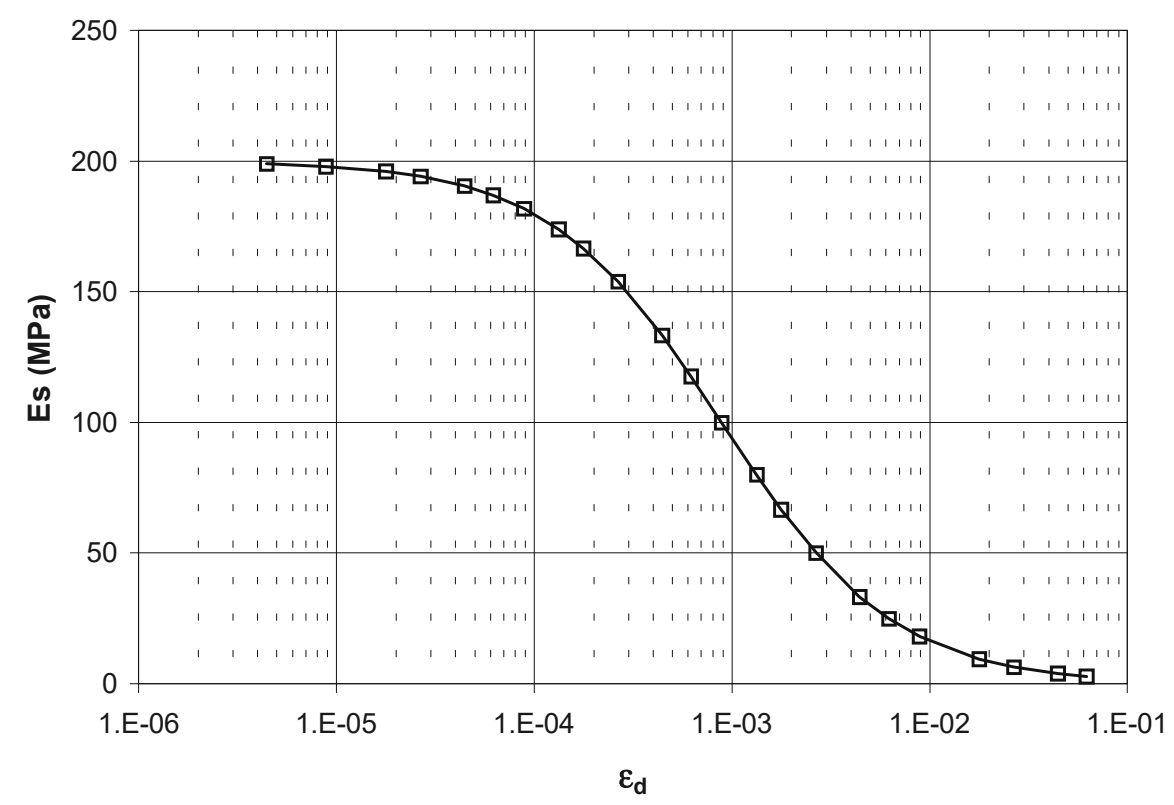

Figure 1. Variation du module sécant avec la déformation déviatorique

La partie élastique linéaire d'une loi élastoplastique a pour vocation à représenter le comportement du sol à l'intérieur de la surface de charge. Dans la pratique, le choix du module sécant dépend du niveau de déformation attendu qui est lui-même 
lié au type d'ouvrage étudié. Pour une fondation superficielle, un niveau de déformation de $0,5 \%$ constitue une hypothèse raisonnable. Sur la courbe en S de la figure 1 , le module sécant correspondant à une déformation de $0,5 \%$ est égal à $30 \mathrm{MPa}$ et on retiendra cette valeur dans la loi élastoplastique utilisée dans cette étude.

\section{Caractéristiques de la loi élastique non linéaire}

Une loi élastique non linéaire permet de prendre en compte naturellement la variation rapide du module sécant avec la déformation. Parmi les nombreuses lois élastiques non linéaires proposées dans la littérature, on a choisi une loi de type hyperbolique qui est définie par l'expression du module d'Young sécant suivante (Hardin, 1972) :

$$
E_{s}\left(\varepsilon_{d}\right)=\frac{E_{0}}{1+\frac{\varepsilon_{d}}{\varepsilon_{r}}}
$$

où :

$\mathrm{E}_{0}$ est le module d'Young maximum (déformations inférieures à $10^{-5}$ );

$\varepsilon_{\mathrm{d}}$ est la déformation déviatorique :

$$
\varepsilon_{d}=\frac{\sqrt{2}}{3}\left[\left(\varepsilon_{1}-\varepsilon_{2}\right)^{2}+\left(\varepsilon_{1}-\varepsilon_{3}\right)^{2}+\left(\varepsilon_{2}-\varepsilon_{3}\right)^{2}\right]^{1 / 2}
$$

$\varepsilon_{\mathrm{r}}$ est une déformation déviatorique de référence.

Le coefficient de Poisson $v$ est constant.

La loi de comportement s'écrit alors en fonction du déviateur des contraintes q :

$$
q=\frac{3 E_{s}\left(\varepsilon_{d}\right)}{2(1+v)} \cdot \varepsilon_{d}
$$

avec :

$$
q=\frac{1}{\sqrt{2}}\left[\left(\sigma_{1}-\sigma_{2}\right)^{2}+\left(\sigma_{1}-\sigma_{3}\right)^{2}+\left(\sigma_{2}-\sigma_{3}\right)^{2}\right]^{1 / 2}
$$


On notera que cette loi hyperbolique ne dérive pas d'un potentiel, ce qui peut conduire à une violation du principe de conservation de l'énergie sur un chemin fermé ; on ne peut donc l'utiliser que sur un trajet de chargement monotone.

Les caractéristiques suivantes ont été calculées à partir de la courbe de la figure 1 :

Module maximal : $\mathrm{E}_{0}=200 \mathrm{MPa}$

Déformation de référence : $\varepsilon_{\mathrm{d}}=8,87.10^{-4}$

\section{Critère de rigidité relative sol-conduite}

On introduit le coefficient de couplage $\mathrm{F}$ et le critère de rigidité RIG que l'on utilisera par la suite pour interpréter les résultats des calculs. Le critère de rigidité résulte d'une comparaison entre la rigidité verticale d'une canalisation et celle d'un prisme de sol de même hauteur et de même largeur.

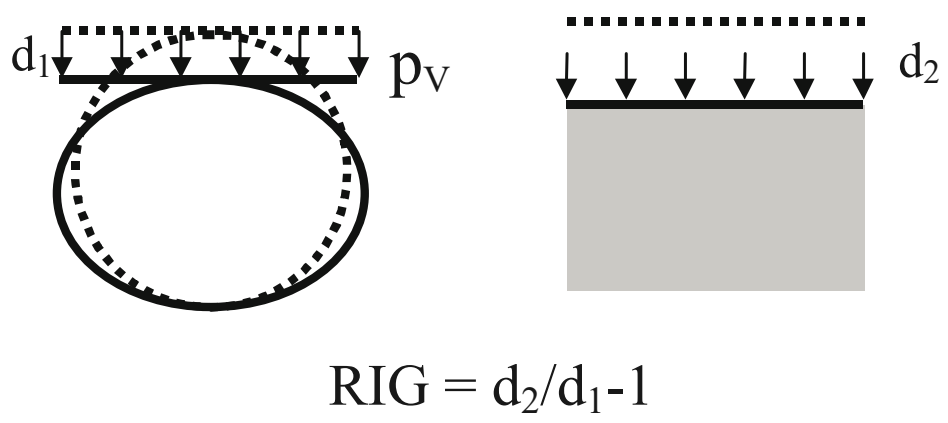

Figure 2. Définition du critère de rigidité RIG

On appel $\mathrm{d}_{1}$ la déformation verticale de la canalisation soumise à une pression verticale uniforme et $\mathrm{d}_{2}$ la déformation du prisme soumise à la même pression (voir figure 2). Le critère de rigidité relative noté RIG est défini par l'expression suivante :

$$
R I G=\frac{d_{2}}{d_{1}}-1=\frac{1}{K_{\alpha} F}-1
$$

où $\mathrm{F}$ est le coefficient de couplage en flexion :

$$
F=\frac{E_{s} R^{3}\left(1-v_{c}^{2}\right)}{E_{c} I_{c}\left(1-v_{s}^{2}\right)}
$$


$\mathrm{K}_{\alpha}$ est un coefficient qui dépend de l'angle d'appui de la conduite et dont la valeur est fixée à $0,1\left(\mathrm{~K}_{\alpha}=1 / 12\right.$ pour $180^{\circ}$ et 0,09 pour $\left.120^{\circ}\right)$.

$R$ est le rayon moyen de la conduite, $E_{s}, v_{s}$ et $E_{c}, v_{c}$ sont respectivement le module et le coefficient de Poisson du sol et de la conduite.

On a par définition :

$$
\begin{array}{lll}
\mathrm{RIG}>0 \text { ou } \mathrm{F}<10 \quad & \Rightarrow & \text { comportement rigide } \\
\mathrm{RIG}<0 \text { ou } \mathrm{F}>10 \quad \Rightarrow & \text { comportement flexible. }
\end{array}
$$

On notera que les sollicitations dans une conduite dépendent fortement du coefficient de couplage en flexion. De manière générale on peut écrire :

$$
S=\frac{A}{1+\alpha F} p_{V}
$$

où $\mathrm{S}$ est une déformation ou un moment par exemple, $\alpha$ est un facteur qui dépend notamment de l'état de l'interface (collée, glissante) et du coefficient de Poisson du sol, et qui n'est pas très différent de $0,1, p_{\mathrm{V}}$ représente la pression verticale transmise par le sol.

\section{Description du modèle aux éléments finis}

On considère un collecteur circulaire en maçonnerie de $2 \mathrm{~m}$ de diamètre extérieur et $20 \mathrm{~cm}$ d'épaisseur, enterré sous une hauteur de sol de $4 \mathrm{~m}$, soit 2 fois le diamètre extérieur. On projette la construction d'une fondation superficielle filante, de largeur $1 \mathrm{~m}$, située à l'aplomb du collecteur et parallèle à son axe longitudinal. On cherche à évaluer l'accroissement de contrainte dans le collecteur apportée par la fondation pour décider d'un éventuel renforcement.

Le matériau du collecteur est, par exemple, une maçonnerie de meulières dont le module homogénéisé a été évalué à $3000 \mathrm{MPa}$. Le matériau du collecteur est modélisé en élasticité linéaire isotrope, le coefficient de Poisson est égal à 0,2.

Le sol est homogène et isotrope. On étudie une loi élastique linéaire, une loi élastoplastique (critère de Tresca) et une loi élastique non linéaire de type hyperbolique. Les caractéristiques mécaniques sont les suivantes :

Loi élastique linéaire :

- module d'Young : $30 \mathrm{MPa}-200 \mathrm{MPa}$;

- coefficient de Poisson : 0,33.

Loi élastoplastique (critère de Tresca) :

- module d'Young : $30 \mathrm{MPa}$;

- coefficient de Poisson : 0,33; 
- cohésion : $70 \mathrm{kPa}$.

Loi élastique non linéaire :

- module d'Young sécant : voir section 3 ;

- coefficient de Poisson : 0,33

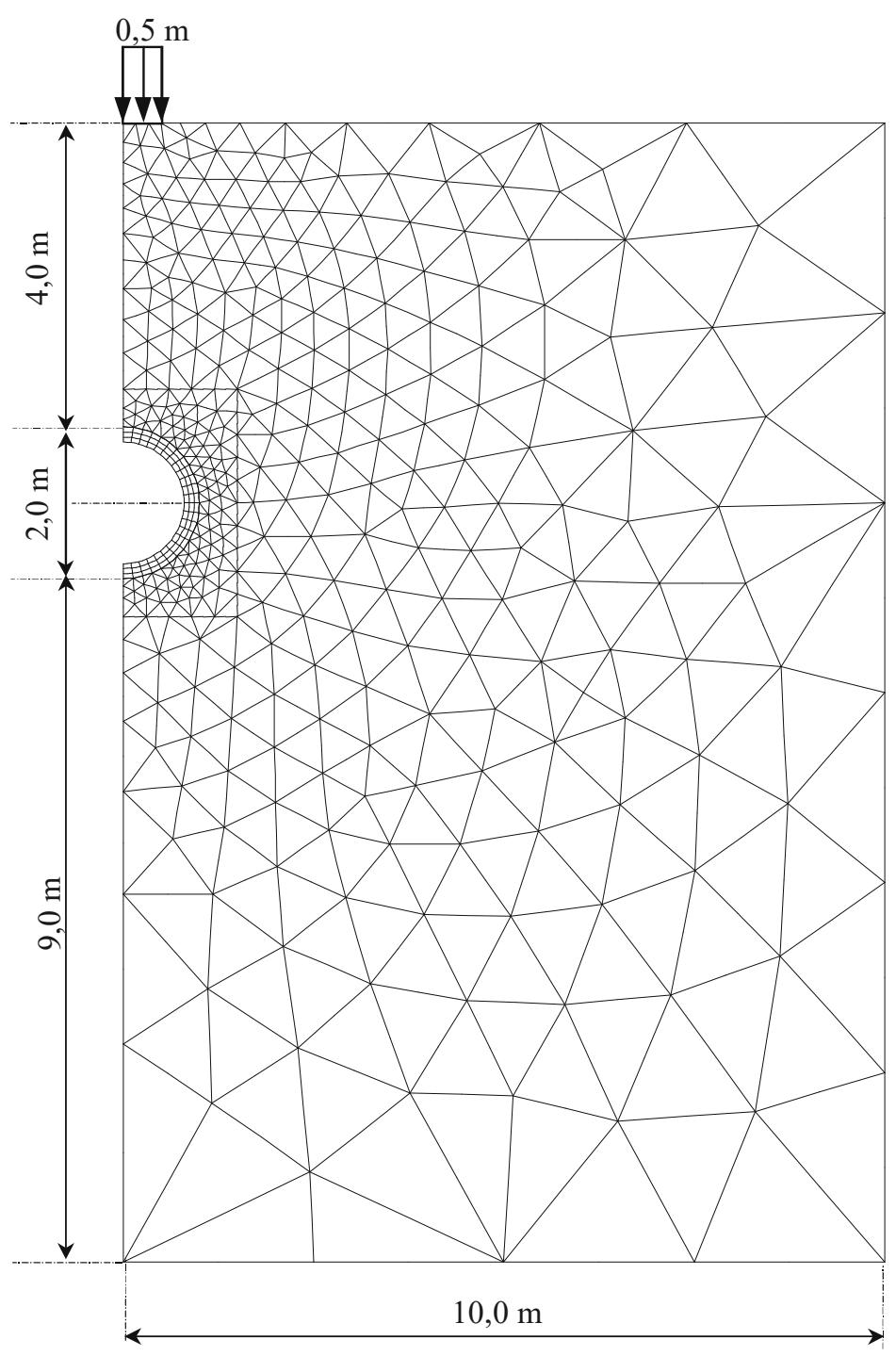

Figure 3. Dessin du maillage

Le chargement est constitué d'une pression uniforme croissante appliquée sur une largeur de $1 \mathrm{~m}$ à la surface du massif (il s'agit donc d'une fondation souple). La bande chargée est axée sur la conduite. Le modèle est traité en déformation plane et les symétries mécaniques et géométriques permettent de ne modéliser que la moitié du modèle physique. La figure 3 représente le maillage utilisé qui comprend 1538 nœuds dont 72 quadrilatères à 8 nœuds pour l'anneau et 623 triangles à 6 nœuds pour le sol. Les limites du maillage ont été définies à une distance égale à 5 fois le diamètre externe de la conduite. Les déplacements verticaux du bord inférieur sont imposés à zéro, ainsi que les déplacements horizontaux des bords latéral gauche et droite. L'interface sol-conduite est parfaitement collée. On notera que les calculs 
avec la loi élasto-plastique ont été réalisés avec le logiciel CESAR-LCPC alors que les calculs avec la loi hyperbolique ont été réalisés avec le logiciel ANSYS.

La cohésion a été choisie de telle manière que les deux lois donnent approximativement la même pression limite de rupture du massif sous une fondation filante. Cette pression limite est donnée pour un sol non pesant et purement cohérent par la formule suivante :

$$
p_{\lim }=(\pi+2) \cdot C=360 \mathrm{kPa}
$$

On notera que le calcul est réalisé sans état initial de contrainte ce qui conduit, pour le critère de plasticité de Tresca, au même résultat qu'un calcul réalisé avec un état géostatique caractérisé par un coefficient de pression des terres au repos $\mathrm{K}_{0}$ égal à 1 . L'étude de l'influence de $\mathrm{K}_{0}$ montre que le déplacement au centre de la fondation est légèrement supérieur ou inférieur selon que $\mathrm{K}_{0}$ est respectivement inférieur ou supérieur à 1 (Mestat, 1994). En revanche, l'état initial n'a pas d'influence sur la valeur de la pression de rupture. L'hypothèse $\mathrm{K}_{0}$ égal 1 serait tout à fait pertinente pour un remblai compacté, par exemple pour une conduite posée sous un remblai routier, en revanche cette valeur serait trop élevé pour un sol normalement consolidé. En tout état de cause, l'état initial a peu d'influence sur les résultats et c'est pour cette raison que l'on a choisi de ne pas en tenir compte.

\section{Résultats des calculs}

\subsection{Résultats du modèle élastique linéaire}

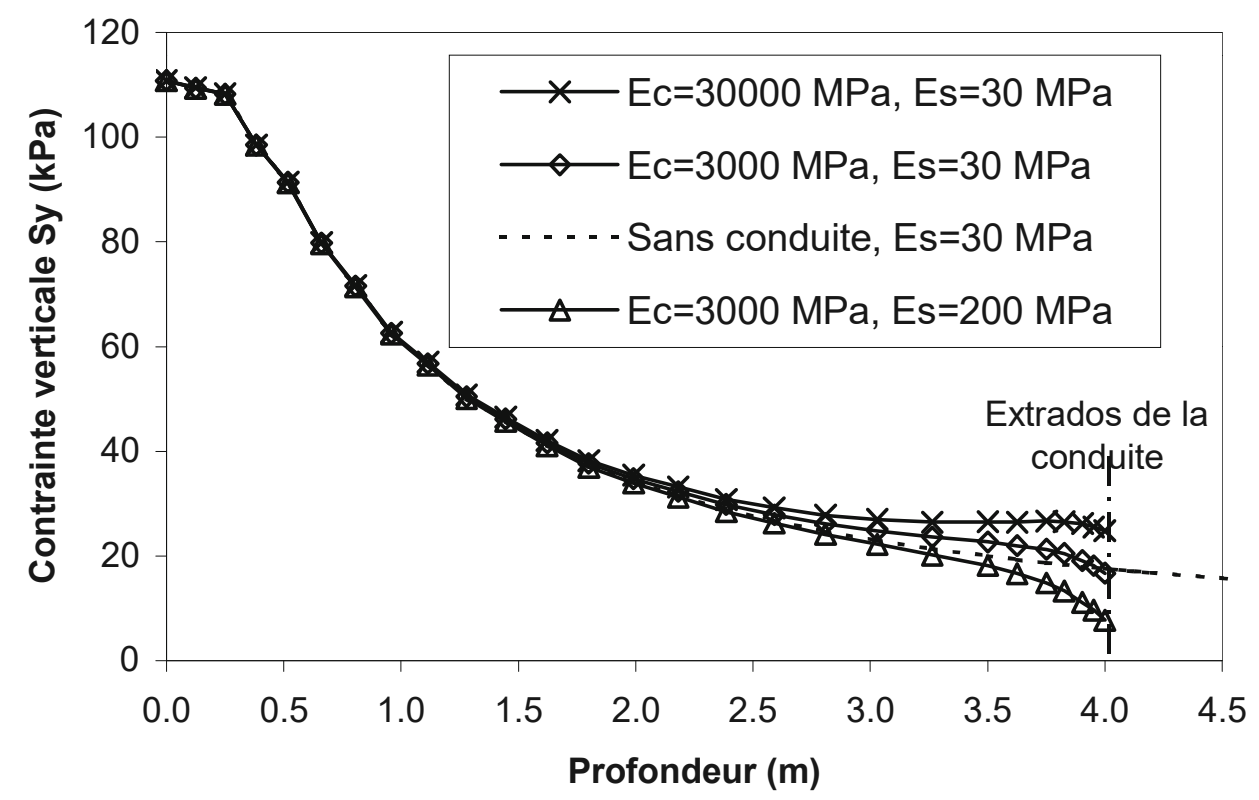

Figure 4. Contrainte verticale dans l'axe du chargement 
On constate sur la figure 4 et le tableau 1, que la contrainte verticale au voisinage de l'extrados de la conduite $(\mathrm{z}=4 \mathrm{~m})$ est supérieure ou inférieure à la contrainte verticale en absence de conduite, selon que le facteur de couplage est respectivement inférieur ou supérieur à 10 ou ce qui est équivalent, selon que la conduite présente un comportement rigide ou flexible.

\begin{tabular}{|c|c|c|c|c|}
\hline Cas n $^{\circ}$ & $\begin{array}{c}\text { Caractéristiques } \\
\text { mécaniques }\end{array}$ & $\begin{array}{c}\text { Facteur de } \\
\text { couplage } \mathrm{F}\end{array}$ & $\begin{array}{c}\text { Type de } \\
\text { comportement }\end{array}$ & $\begin{array}{c}\text { Contrainte verticale à } \\
\mathrm{z}=4 \mathrm{~m}(\mathrm{kPa})\end{array}$ \\
\hline 1 & $\begin{array}{c}\mathrm{Ec}=3000 \mathrm{MPa} \\
\mathrm{Es}=30 \mathrm{MPa}\end{array}$ & 11,8 & neutre & 16,7 \\
\hline 2 & $\begin{array}{c}\mathrm{Ec}=3000 \mathrm{MPa} \\
\mathrm{Es}=200 \mathrm{MPa}\end{array}$ & 78,6 & flexible & 7,7 \\
\hline $3^{*}$ & $\begin{array}{c}\mathrm{Ec}=30000 \mathrm{MPa} \\
\mathrm{Es}=200 \mathrm{MPa}\end{array}$ & 1,18 & rigide & 24,8 \\
\hline 4 & Sans conduite & Sans objet & Sans objet & 17,6 \\
\hline
\end{tabular}

* Le cas $n^{\circ} 3$ a été ajouté pour la comparaison

Tableau 1. Résultats des calculs élastiques linéaires

La figure 5 montre que, pour une même pression appliquée égale à $100 \mathrm{kPa}$, l'ovalisation verticale $\Delta_{\mathrm{V}}$ de la conduite diminue rapidement avec l'augmentation du facteur de couplage $\mathrm{F}$. La figure 5 montre également que le comportement neutre $(\mathrm{F}=10)$ correspond à un point d'inflexion de la courbe et à une diminution de l'ordre de $50 \%$ de l'ovalisation maximale que l'on peut extrapoler pour $\mathrm{F}=0$.

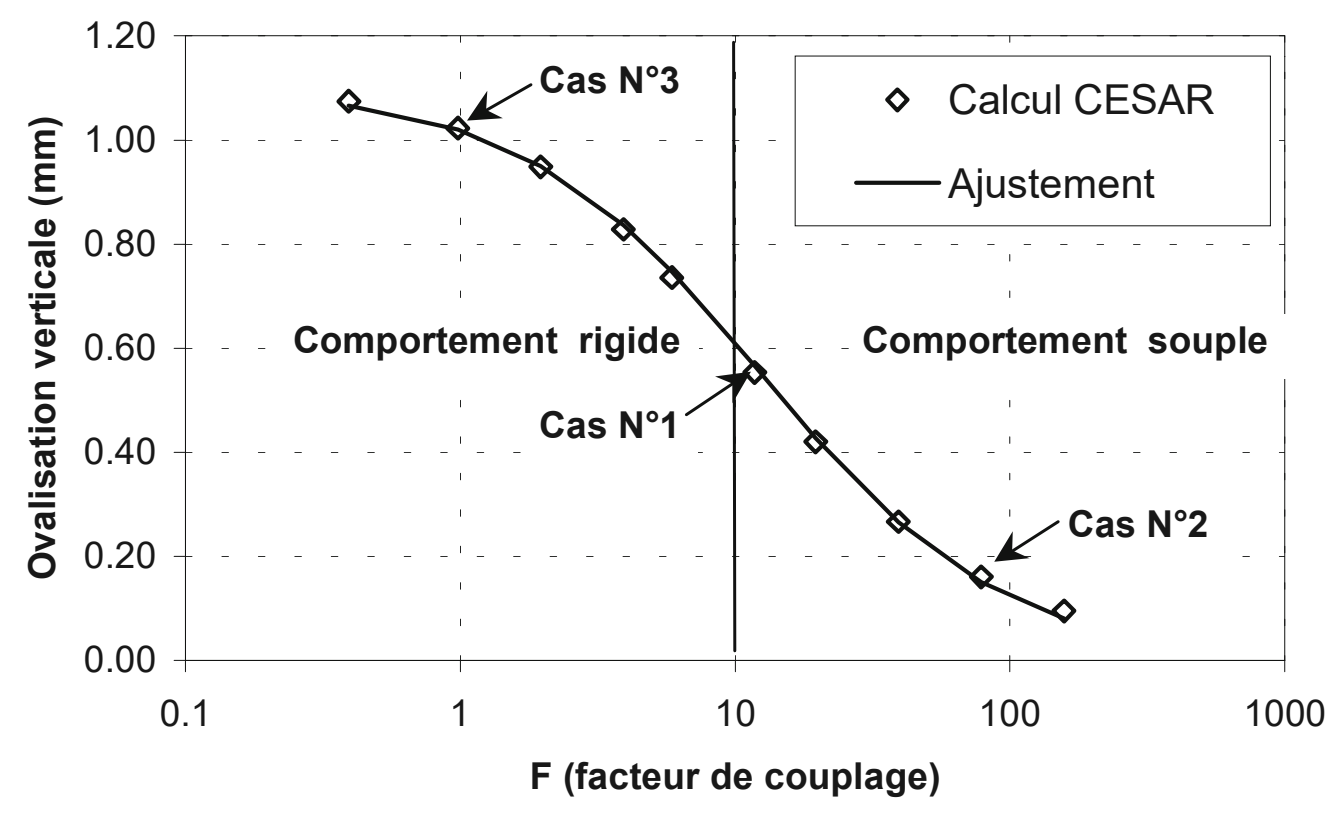

Figure 5. Evolution de l'ovalisation verticale en fonction du facteur de couplage 
L'ajustement de la formule [7] sur les résultats des calculs MEF donne le résultat suivant ( $\mathrm{p}$ est la pression sous la fondation exprimée en $\mathrm{MPa}$ ) :

$$
\Delta_{V}(m m)=\frac{11 p(M P a)}{1+0,08 F}
$$

A partir des formules [9] et [6], on peut exprimer le module du sol en fonction de l'ovalisation verticale de la conduite :

$$
E_{s}(M P a)=31,8\left[\frac{11 p}{\Delta_{V}}-1\right]
$$

La formule [10] définie le module élastique linéaire « équivalent » du sol.

\subsection{Résultats du modèle élastoplastique}

La figure 6 représente à gauche les courbes d'isovaleurs de la déformation déviatorique pour une charge égale à $50 \%$ de la charge de rupture et à droite l'évolution de l'isovaleur $0,1 \%$ en fonction du facteur de charge Fc (\% de la charge de rupture). On constate que la zone des déformations supérieures à $0,1 \%$ (en grisé) atteint une profondeur de l'ordre de 3,5 m soit 3,5 fois la largeur de la fondation. Le reste du massif, et en particulier le sol encaissant la conduite, est donc dans un état de petites déformations. On notera que le massif n'est pas plastifié à la moitié de la charge critique et que la répartition des contraintes est donc identique au cas élastique linéaire.

On constate également que, pour un facteur de charge de $30 \%$ (valeur typique d'une charge de service compte tenu des coefficients de sécurité habituels, voir Frank, 1999), l'étendue de la zone des déformations supérieures à $0,1 \%$ se limite à une profondeur de l'ordre de 1,5 fois la largeur de la fondation filante. Le niveau de déformation de la plus grande partie du massif de sol autour de la conduite est donc sensiblement inférieur à $0,1 \%$. Par conséquent, pour modéliser le comportement de la conduite et son interaction avec le sol, il est souhaitable d'utiliser un module d'Young représentatif de la gamme de déformation $0-0,1 \%$, qui est celle où le module sécant chute très rapidement d'un ordre de grandeur par rapport au module maximal (qui correspond à une déformation inférieure à $10^{-5}$ ). Le module sécant de $30 \mathrm{MPa}$ utilisé dans ce calcul et qui correspond à un niveau de déformation supérieur à $0,1 \%$, est bien adapté à la modélisation de la fondation, mais trop faible pour la modélisation de l'interaction sol-conduite. 

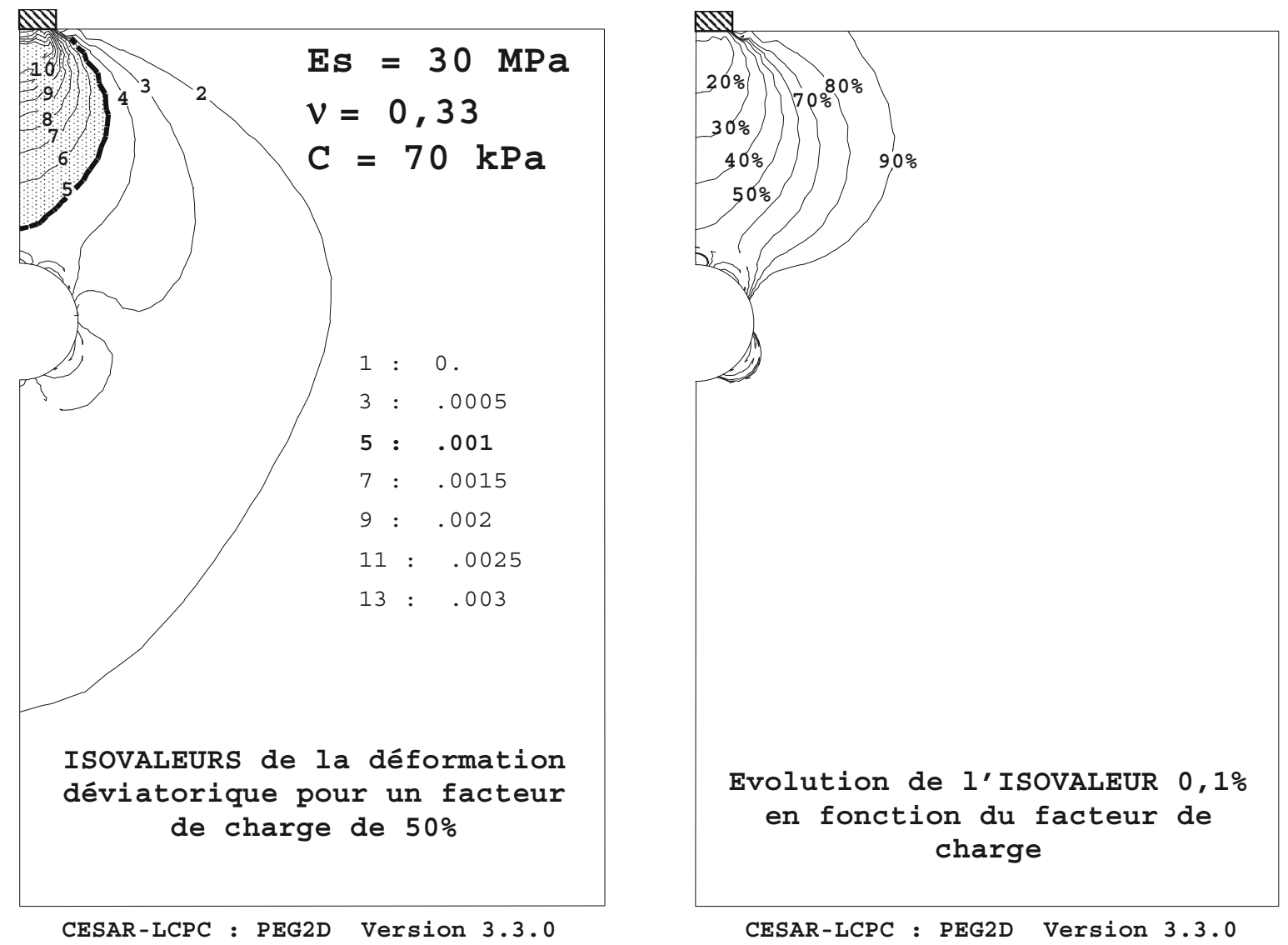

Figure 6. Isovaleurs de la déformation déviatorique en fonction du facteur de charge

\subsection{Résultats obtenus avec le modèle hyperbolique}

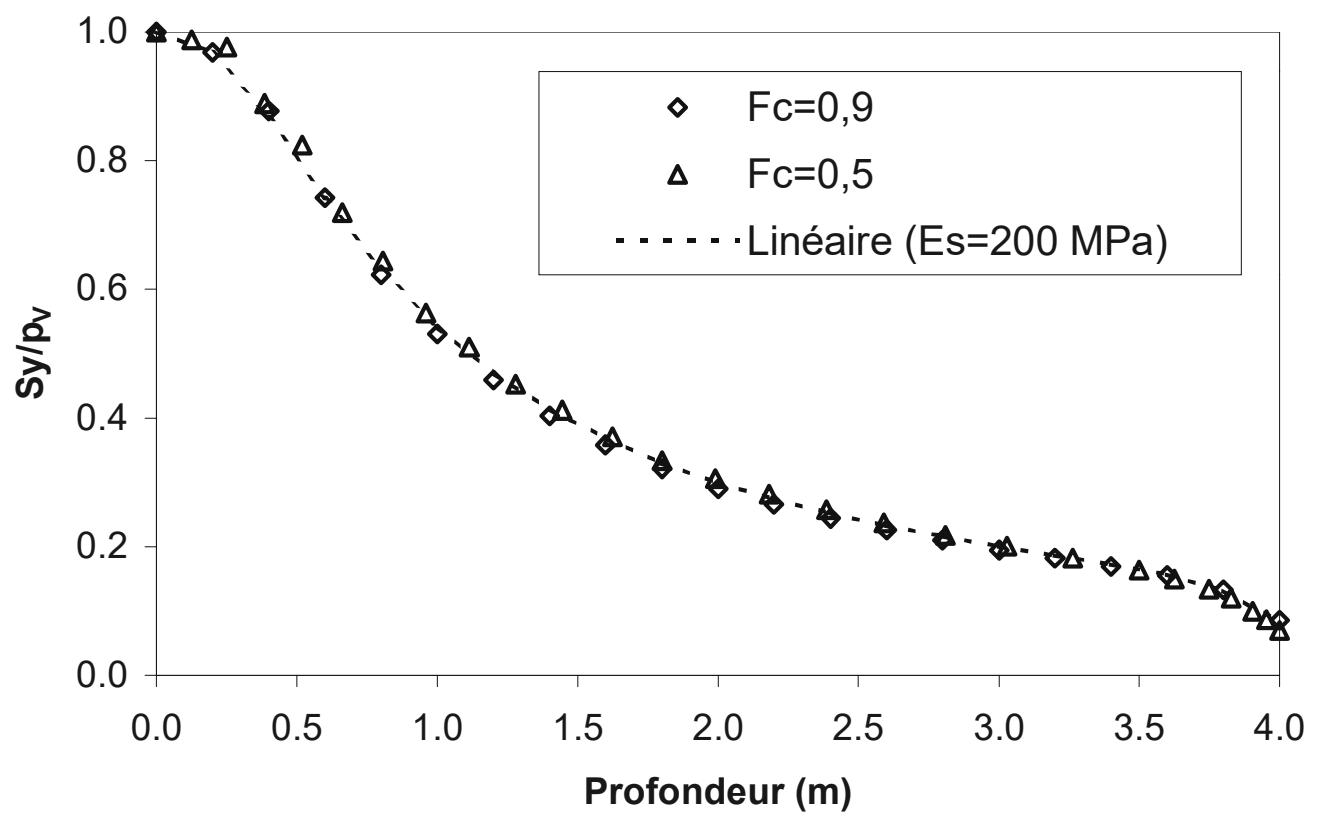

Figure 7. Contrainte verticale réduite dans l'axe du chargement 
On constate sur la figure 7 que l'évolution de la contrainte verticale dans l'axe du chargement en fonction de la profondeur est peu sensible au facteur de charge et très proche de la solution élastique linéaire. Le comportement élastique non linéaire a donc une influence négligeable sur la distribution des contraintes dans le sol par rapport à un comportement élastique linéaire.

La figure 8 montre que le module sécant diminue rapidement sous la fondation avec l'augmentation du chargement alors qu'il reste pratiquement égal au module maximum $\mathrm{E}_{0}(200 \mathrm{MPa}$ ) au voisinage de la canalisation (à une profondeur de $4 \mathrm{~m}$ ).

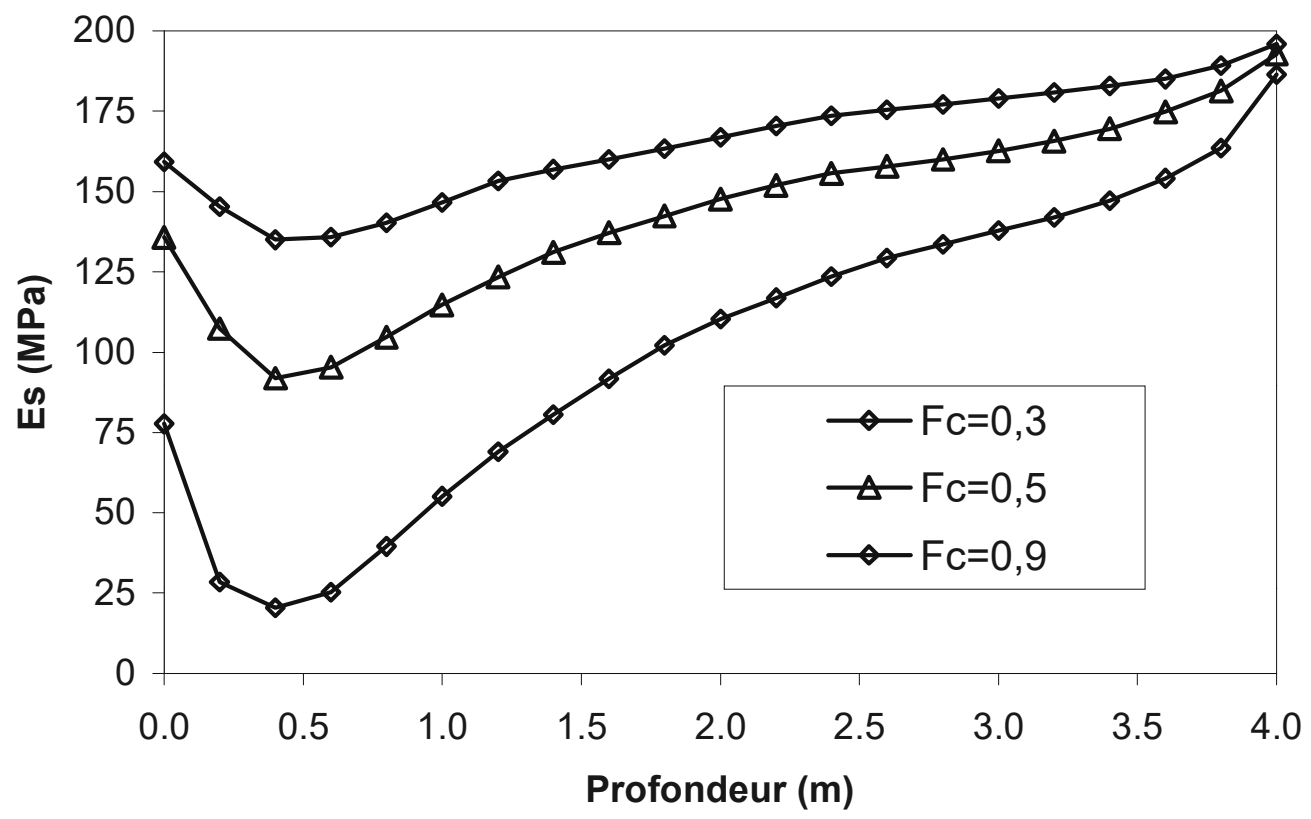

Figure 8. Evolution du module sécant avec la profondeur et le facteur de charge

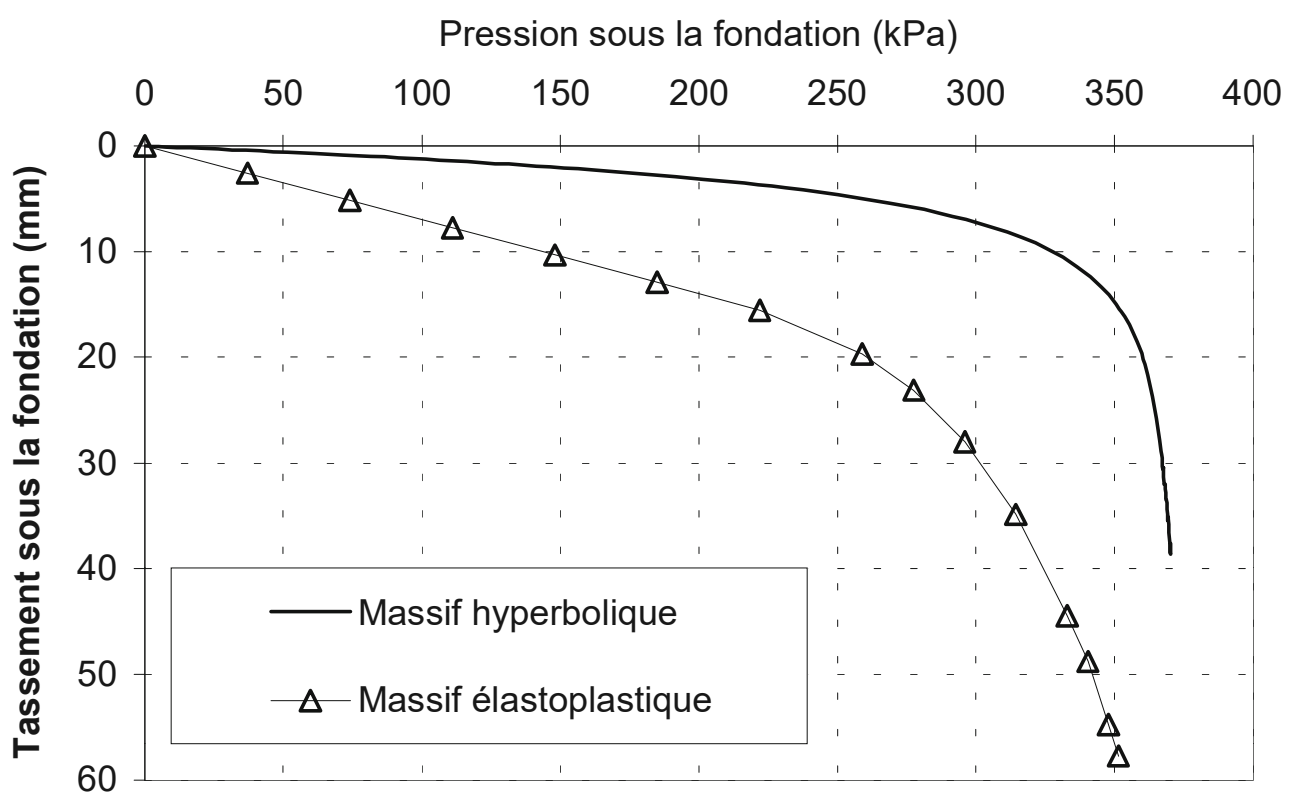

Figure 9. Courbes de tassement de la fondation 
La figure 9 représente la variation du déplacement vertical au centre de la fondation avec la pression appliquée, pour les deux lois hyperbolique et élastoplastique. Les courbes divergent à l'approche d'une pression de l'ordre de $370 \mathrm{kPa}$. La courbe hyperbolique est plus raide que la courbe élastoplastique, surtout au début du chargement.

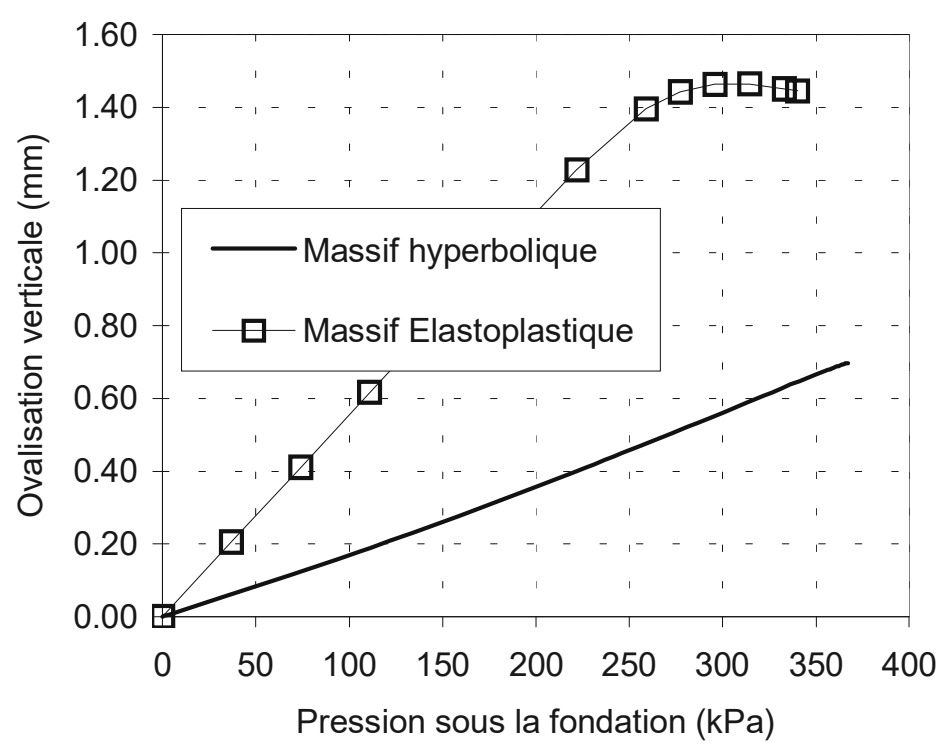

Figure 10. Variation de l'ovalisation verticale de la conduite en fonction de la pression appliquée par la fondation

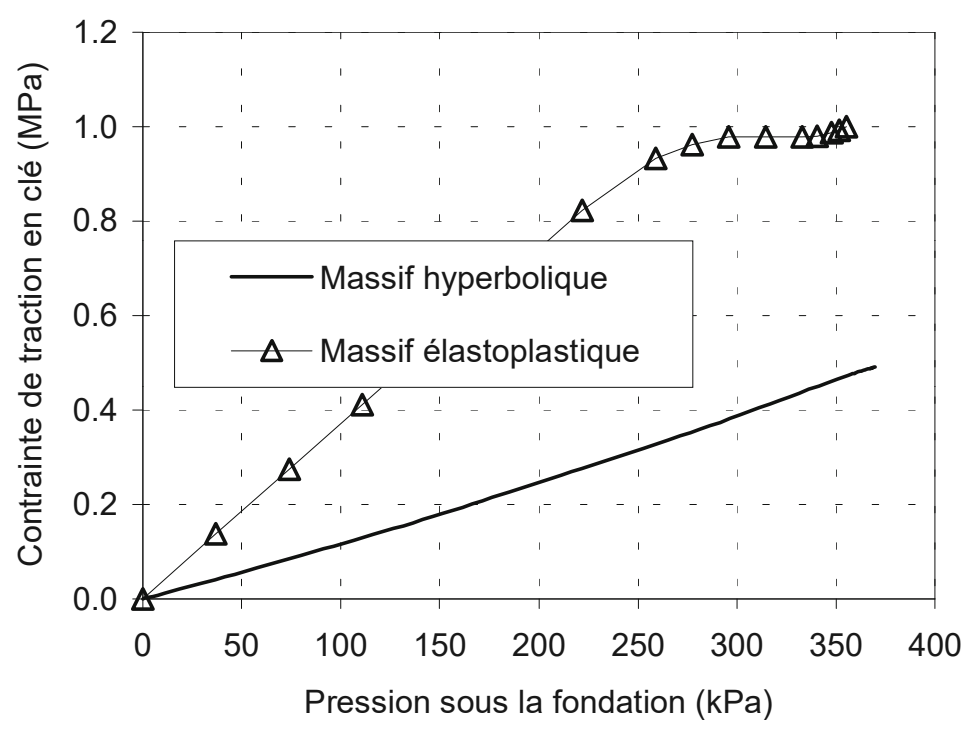

Figure 11. Variation de la contrainte de traction maximale dans la conduite en fonction de la pression appliquée par la fondation

Les figures 10 et 11 représentent respectivement l'évolution de l'ovalisation verticale (raccourcissement du diamètre vertical) de la conduite et l'évolution de la 
contrainte maximale de traction (située en clé à l'intrados) de la conduite, en fonction de la pression appliquée par la fondation. On constate que les résultats de la loi hyperbolique sont pratiquement 3 fois plus faibles que ceux de la loi élastoplastique. Cette différence est simplement due à l'interaction sol-conduite qui profite de la raideur plus élevée du massif aux petites déformations comme on l'a vu dans l'analyse des résultats du modèle élastique linéaire.

La figure 12 montre que le module élastique linéaire équivalent (formule [10]) décroît faiblement avec la pression appliquée en surface. Au point de rupture de la fondation, sa valeur est égale à $165 \mathrm{MPa}$ soit $80 \%$ du module maximum.

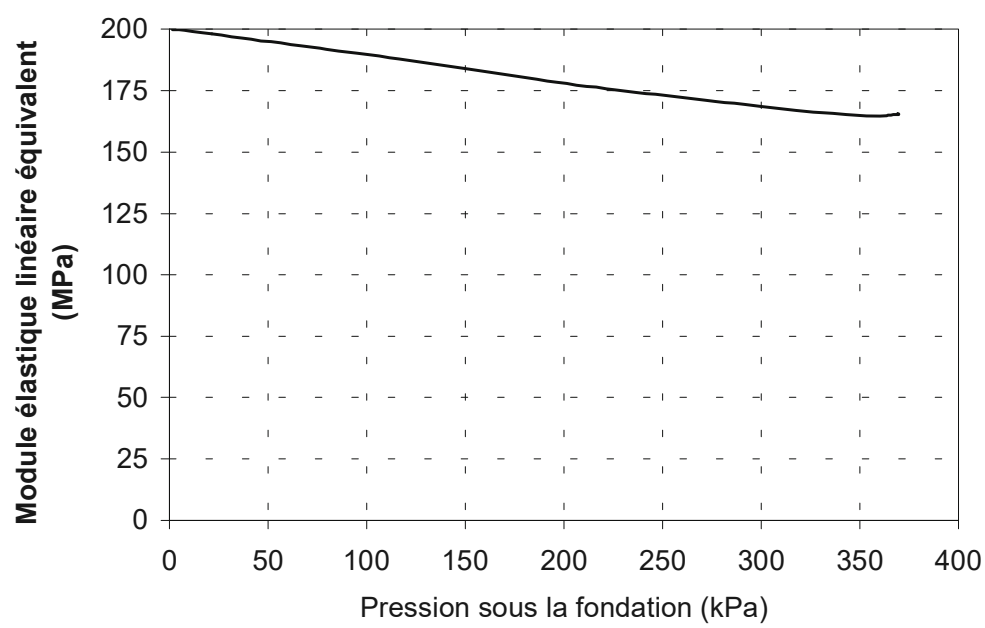

Figure 12. Module élastique équivalent pour la conduite en fonction de la pression appliquée sous la fondation

\section{Conclusions}

A partir des résultats exposés précédemment, on peut faire les remarques suivantes :

Les sollicitations dans la conduite dépendent fortement du coefficient de couplage en flexion qui mesure l'intensité de l'interaction sol-structure (figure 5) et donc le module du sol pour une conduite avec des caractéristiques mécaniques données.

Le calcul élastoplastique avec un module linéarisé sur la plage 0-0,5\% montre que pour une charge inférieure à $50 \%$ de la charge critique, la plus grande partie du massif n'est pas plastifié et reste dans un état de petites déformations (figure 6). Par conséquent, le choix du module linéarisé (ou de la plage de linéarisation) est important pour le comportement du massif en dehors de la zone d'influence directe de la fondation.

La variation du module sécant avec la déformation (comportement élastique non linéaire) n'a pratiquement pas d'influence sur la distribution des contraintes dans le 
massif de sol (la distribution reste très proche du cas élastique linéaire). En revanche, le module sécant du sol varie avec la profondeur et l'intensité du chargement ce qui implique que le champ de déformation est très différent du cas élastique linéaire. Dans la zone d'influence de la fondation, le module sécant diminue rapidement avec le chargement alors que dans la zone éloignée autour de la conduite le module sécant reste proche de sa valeur maximale (figure 8). La conduite "voit» donc un sol nettement plus raide que la fondation, ce qui est impossible à modéliser avec un module constant.

Le choix d'un module de sol adapté à la modélisation de la fondation (quelle que soit la plage de linéarisation retenue) conduit à sous-estimer la raideur du sol autour de la conduite et, par conséquent, à surestimer les contraintes et déplacements dans la conduite (figures 10 et 11). La diminution des sollicitations dans la conduite dans le cas non linéaire, provient de la dépendance du module avec la déformation et non pas d'une distribution de contrainte différente sous la fondation.

Comme le calcul élastoplastique est conservateur pour la conduite, on peut effectivement conserver les résultats obtenus avec $30 \mathrm{MPa}$ pour la fondation et ceux correspondant à $200 \mathrm{MPa}$ (linéaire et non linéaire) pour la conduite. Mais une loi élastique non linéaire éventuellement couplée à un critère plastique est indubitablement plus satisfaisante car elle permet de prendre en compte la variation de la raideur du sol avec la déformation qui est une propriété fondamentale des sols notamment dans les études d'interaction sol-structure.

\section{Bibliographie}

Burland J. B., "Small is beautiful - the stiffness of soils at small strains", Can. Geotech. J. vol. 26, 1989, p. 499-516.

Frank R., «Calcul des fondations superficielles et profondes », Techniques de l'Ingénieur (TI), Presses de l'ENPC, 1999.

Hardin B. O., Drnevich V. E., "Shear modulus and damping in soil: design equation and curves", Journal of Geotechnical Engineering Division, ASCE, vol. 98, SM7, 1972, p. 667-691.

Homsi M., Contribution à l'étude des propriétés mécaniques des sols en petites déformations à l'essai triaxial, Thèse de Doctorat, Ecole Centrale de Paris, 1986.

Jardine R. J., Potts D. M., Fourie A. B., Burland J. B., "Studies of the influence of non linear stress-strain characteristics in soil-structure interaction", Géotechnique, vol. 36, n 3 , 1986, p. 377-396.

Mestat P., «Validation du progiciel CESAR-LCPC en comportement mécanique non linéaire. Volume 1: Fondations superficielles et tunnels », Série géotechnique, GT 58, 1994.

Thépot O., Prise en compte des caractéristiques en petites déformations des sols dans l'étude du comportement des collecteurs enterrés, Thèse de Doctorat, Ecole Nationale des Ponts et Chaussées, 2004. 